EPJ Web of Conferences 85, 02036 (2015)

DOI: $10.1051 /$ epjconf/ 20158502036

(C) Owned by the authors, published by EDP Sciences, 2015

\title{
Polarised Drell-Yan measurements at COMPASS
}

\author{
Michela Chiosso ${ }^{1,2, a}$ on Behalf of the COMPASS Collaboration \\ ${ }^{1}$ University of Torino \\ ${ }^{2}$ INFN, Sezione di Torino
}

\begin{abstract}
Much of the information that exists today about Transverse Momentum Dependent Parton Distribution Functions (TMDs) comes from SIDIS measurements with unpolarised and polarised beams and targets where they appear convoluted with fragmentation functions (FFs). Drell-Yan (DY) measurements are complementary to those by SIDIS experiments, as they allow to measure convolutions of only Parton Distribution Functions (PDFs) without involving FFs. Moreover, given the T-odd character of both Sivers and Boer-Mulders functions, the sign of these TMDs is expected to be reversed when observed from SIDIS or from DY. Measurements of SIDIS were performed by Compass in the period 2002 to 2007 and in 2010, using a naturally polarised $\mu^{+}$beam and a solid state target polarised either longitudinally or transversely with respect to the beam direction. Now the COMPASS Experiment has the unique opportunity to access TMDs from single-polarised Drell-Yan processes as well, in the same kinematical domain of the SIDIS data and with the same experimental setup; it will allow to test the TMDs universality and the sign change prediction for the first time. We present here the upcoming polarised DY measurements at COMPASS .
\end{abstract}

\section{Drell-Yan vs SIDIS}

The main goal of COMPASS DY programme [2] is to measure the process $\pi^{-} p^{\uparrow} \rightarrow \mu^{+} \mu^{-} X$ with a $190 \mathrm{GeV} \pi^{-}$ beam scattered off a transversely polarised proton target $\left(\mathrm{NH}_{3}\right)$.

By performing the measurements of target spin (in)dependent asymmetries in DY reaction and comparing the results with ones obtained in SIDIS, we will be able for the first time ever to verify the universality of TMD approach for the description of these reactions. This would be a crucial test of QCD in the non-perturbative regime. In Fig. 1 the $Q^{2}$ versus $x$ phase-space region ex-

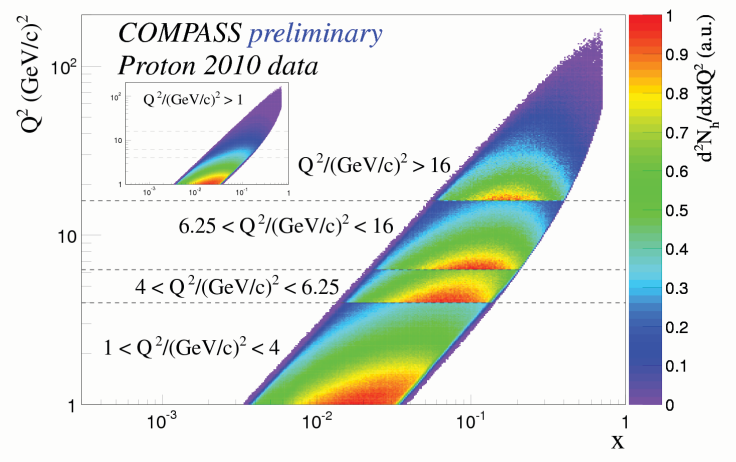

Figure 1. $Q^{2}$ versus $x$ distribution, from Drell-Yan Monte-Carlo events with $4 \leq M_{\mu \mu} \leq 9 \mathrm{GeV} / \mathrm{c}^{2}$ and from 2010 SIDIS data with transversely polarized $\mathrm{NH}_{3}$ target.

\footnotetext{
ae-mail: chiosso@to.infn.it
}

pected to be covered by the COMPASS DY measurements is superimposed with the one accessed by the SIDIS processes at COMPASS with transversely polarized $\mathrm{NH}_{3}$ target (2010 data). So we expect good overlap of the phase-space regions for DY and SIDIS. $Q^{2}$ is the dimuon invariant mass squared, and $x$ stands for the momentum fraction of the annihilated quark(antiquark) from the target nucleon.

In Fig. 2 the results of the Sivers asymmetry extraction from COMPASS 2010 SIDIS data, in the $Q^{2}$ ranges covered by COMPASS DY, are shown for $z>0.2$, as function of $x, z, P_{T}$ and $W$, for positive and negative unidentified hadrons. $z$ is fraction of the energy of the virtual photon carried by hadron, $P_{T}$ is the hadron transverse momentum with respect to the virtual photon, $W$ is the mass of the final hadronic state. The minimum $\mathrm{z}$ value has been set to 0.2 to select hadrons from the current fragmentation region. A clear Sivers asymmetry is visible in all $Q^{2}: \mathrm{x}$ range for positive hadrons. A non-zero effect is also observable for negative hadrons at high $Q^{2}$ :x values.

\section{Single-polarized DY asymmetries in LO QCD parton model}

The general form for the Drell-Yan cross section with polarised nucleons in the initial state (expressed in the azimuthal angle $\phi_{S}$ of the target polarisation vector in the target rest frame, and the polar and azimuthal angles $\theta$ and $\phi$ of the lepton momentum in the Collins-Soper frame ) can be found in Ref. [1]. In the COMPASS case, where only the target nucleon is polarised, four non-vanishing a- 


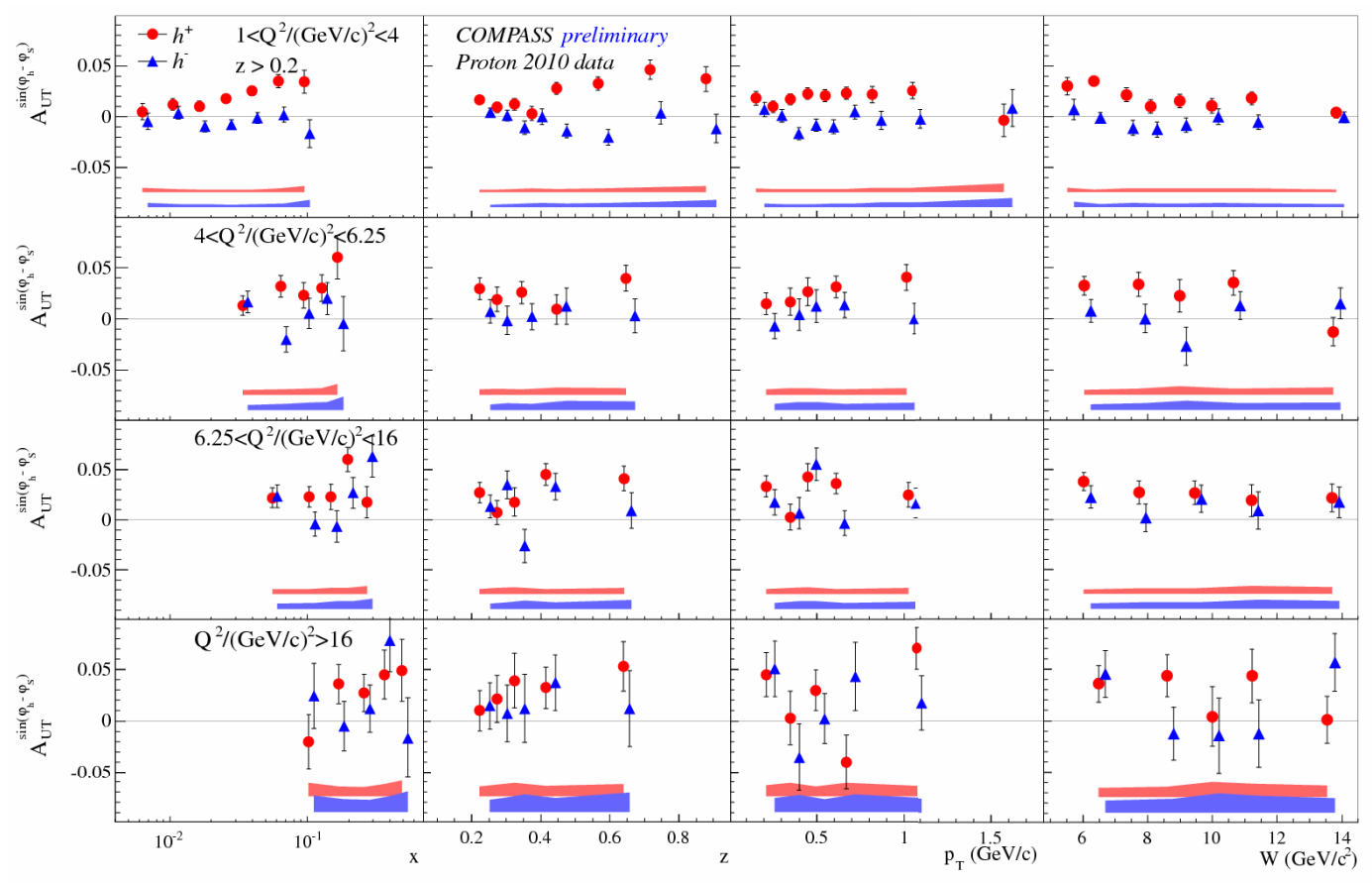

Figure 2. Sivers asymmetry $A_{U T}^{\sin \left(\phi_{S}\right)}$ extraction from COMPASS 2010 SIDIS data for $\mathrm{z}>0.2$

zimuthal asymmetries at LO gives us an input on different convolutions of PDFs and TMDs:

- $A_{U}^{\cos 2 \phi}$ gives access to the Boer-Mulders functions, $h_{1}^{\perp}$, of incoming hadrons,

- $A_{T}^{\sin \phi_{S}}$ to the Sivers function, $f_{1 T}^{\perp}$, of the target nucleon,

- $A_{T}^{\sin \left(2 \phi+\phi_{S}\right)}$ to the Boer-Mulders functions of beam hadron and to the pretzelosity function, $h_{1 T}^{\perp}$, of the target nucleon,

- $A_{T}^{\sin \left(2 \phi-\phi_{S}\right)}$ to Boer-Mulders functions of beam hadron and to the transversity function, $h_{1}$, of the target nucleon.

To disentangle the PDFs in each of these asymmetries requires certain knowledge of other PDFs. The simplest case is the extraction of the Sivers function, which is convoluted with the well known pion unpolarised PDF. More complex is to access the transversity and pretzelosity of the nucleon for which the knowledge of the Boer-Mulders function of the pion is needed. There are two different scenarios to evaluate this function:

1. measure the $A_{T}^{\sin \left(2 \phi-\phi_{S}\right)}$ asymmetry and use the parametrization of $h_{1}$ from Refs. [3, 4] to extract Boer-Mulders function of the pion;

2. use the already existing measurements of $A_{U}^{\cos 2 \phi}$ and the information on the Boer-Mulders function of the target nucleon. This asymmetry was measured in the unpolarized $\pi+N$ Drell-Yan process by the NA10 and E615 Collaborations [5-7] and more recently by the E866/NuSea Collaboration at FNAL $[8,9]$ in $p+N$ Drell-Yan reaction.

\section{Expected event rate and statistical precision}

A full Monte-Carlo simulation was performed to evaluate the COMPASS spectrometer acceptance in the invariant mass intervals $4 \leq M_{\mu \mu} \leq 9 \mathrm{GeV} / \mathrm{c}^{2}$ and $2 \leq M_{\mu \mu} \leq 2.5$ $\mathrm{GeV} / \mathrm{c}^{2}$. These two regions are considered to be the best mass intervals for Drell-Yan analysis, avoiding the large combinatorial background that shall dominate at lower dimuon masses, and excluding the $\phi, J / \psi$ and $\Upsilon$ vectormeson resonances. The first (high) mass region certainly provides a cleaner sample of DY events, because of the very small contribution from uncorrelated combinatorial muon background (originating from pion and kaon decays) and open-charm semileptonic decays (i.e $D \bar{D}$ decays into muons). It is particularly interesting, as it covers mostly the region of valence quarks, but the DY crosssection for these masses is almost a factor 5 smaller than in the second (intermediate) mass region. Nevertheless, with the foreseen increased beam intensity of $10^{8}$ particles/s we can achieve a luminosity of $2.3 \times 10^{33} \mathrm{~cm}^{-2} \mathrm{~s}^{-1}$ and we expect a DY event rate of about 2034 events/day for the high mass region. In Fig. 3 the expected statistical error of the Sivers asymmetry, assuming one year of data taking, are shown. The expected statistical errors in all the four LO azimuthal asymmetries are as given in table 1 .

As the measurement is statistics limited, the luminosity optimisation is mandatory. The installation of an hadron absorber downstream of the target allows to reduce the high secondary particle flux produced by the interaction of the pion beam in the target and, consequently, the 

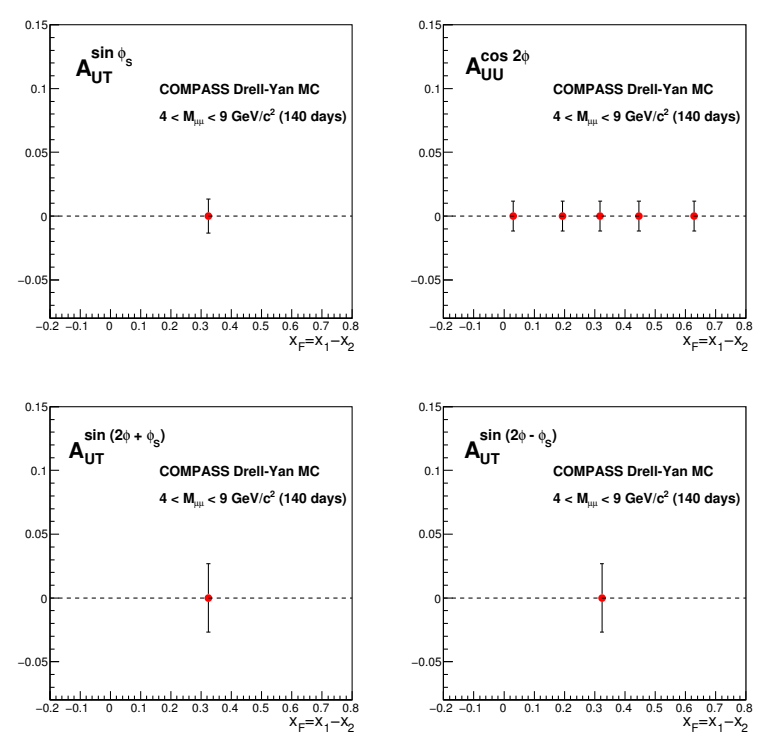

Figure 3. Expected statistical error of the Sivers asymmetry in the dimuon mass range $4 . \leq M_{\mu \mu} \leq 9 . \mathrm{GeV} / \mathrm{c}^{2}$, assuming one year of data taking (140 days).

Table 1. Expected statistical errors in the asymmetries, in 140 days of data-taking, with a beam momentum of $190 \mathrm{GeV} / \mathrm{c}$.

\begin{tabular}{ll}
\hline Dimuon mass $\left(\mathrm{GeV} / \mathrm{c}^{2}\right)$ & $4<M_{\mu \mu}<9$ \\
\hline$\delta A_{U}^{\cos 2 \phi}$ & 0.005 \\
\hline$\delta A_{T}^{\sin \phi_{S}}$ & 0.013 \\
\hline$\delta A_{T}^{\sin \left(2 \phi+\phi_{S}\right)}$ & 0.027 \\
\hline$\delta A_{T}^{\sin \left(2 \phi-\phi_{S}\right)}$ & 0.027 \\
\hline
\end{tabular}

tracking detector occupancies. This makes an increase in the intensity of the incident pion beam possible. The absorber, $236 \mathrm{~cm}$ long, is made by alumina $\left(\mathrm{Al}_{2} \mathrm{O}_{3}\right)$, with a $140 \mathrm{~cm}$ long tungsten beam plug inside it to stop the noninteracted beam. The alumina absorber stops the secondary particles flux, minimising multiple scattering; this is essential to disentangle the two oppositely polarised target cells in the track reconstruction.

A scintillating fibers vertex detector has been placed between the target and the absorber, in order to further improve the resolution in the vertex position reconstruction and, consequently, the two target cells separation.

\section{Feasibility of the measurement}

In order to study the feasibility of the proposed polarised Drell-Yan programme and to evaluate in a realistic way the achieavable statistical accuracies, short beam tests were performed in 2007, 2008, 2009 and 2012. During the last one a prototype hadron absorber, made of concrete and stainless steel, 1 meter long each, and with a tungsten beam plug in its center, was installed downstream of the target. A $\pi^{-}$beam with momentum $190 \mathrm{GeV} / \mathrm{c}$ was used. The beam intensity was $8 \times 10^{7}$ pions/spill (spill length of $9.6 \mathrm{~s}$ ), except for two runs when it was eventually increased to $1.5 \times 10^{8}$ pions/spill. The obtained dimuon mass spectrum is shown in Fig. 4. The number of found $J / \psi, 6787 \pm$ 109 , is in agreement with the Monte-Carlo simulations. The spectrum was fitted from 1.5 to $5.0 \mathrm{GeV} / \mathrm{c}^{2}$, using two Gaussians and two exponential functions. The first Gaussian corresponds to the $\mathrm{J} / \psi$ and the second one to the $\psi^{\prime}$.The number of fitted $\psi$ ' is $146 \pm 36$ which is in good agreement with the expectation that the number of $\psi^{\prime}$ is about $2 \%$ of the number of $\mathrm{J} / \psi$. The continuum under the $\mathrm{J} / \psi$ peak includes the contribution from Drell-Yan, the combinatorial background and the open charm background. The $x_{1}$ (x pion) versus $x_{2}$ (x proton) distribution

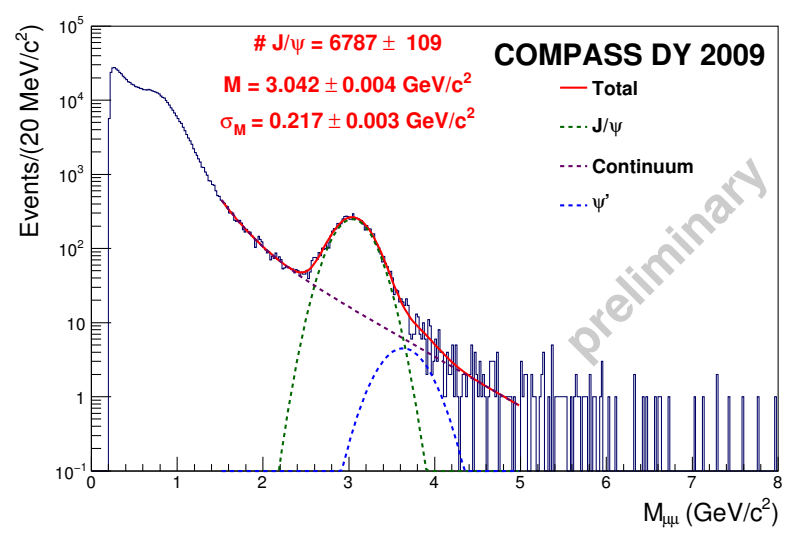

Figure 4. The measured $\mu^{+} \mu^{-}$invariant mass distribution.

for dimuon masses larger than $2.5 \mathrm{GeV} / c^{2}$ is visible in Fig. 5. It shows a good acceptance in the valence quark region.

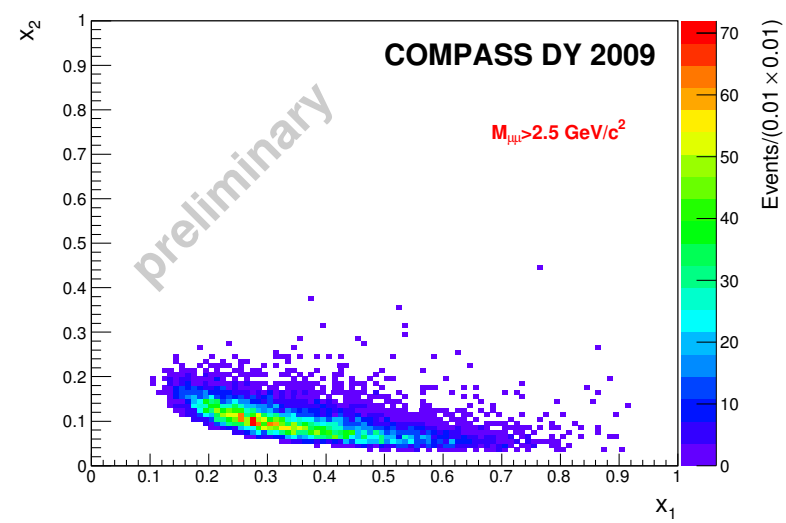

Figure 5. The measured $x_{1}$ versus $x_{2}$ distribution for dimuon masses larger than $2.5 \mathrm{GeV} / c^{2}$

\section{Conclusions}

The COMPASS DY programme is starting in October 2014, with a two months-long pilot run. Physics data taking will continue one whole year run (about 140 days) during 2015. We have the unique opportunity to study the transversity and TMDs of the nucleon with a measurement complementary to the SIDIS one so far performed by 
COMPASS . By measuring the target-spin-(in)dependent asymmetries in the Drell-Yan reaction and comparing the results with the ones obtained in SIDIS we will be able to verify the universality of the TMD approach for the description of these reactions and to test the sign-change prediction of the Sivers and the Boer-Mulders function for the first time. The expected statistical error of the Sivers asymmetry in the dimuon mass range $4 . \leq M_{\mu \mu} \leq 9$. $\mathrm{GeV} / \mathrm{c}^{2}$, in one year of data taking (140 days), is $1.3 \%$; it is $0.5 \%$ for the Boer-Mulders asymmetry.

\section{References}

[1] S. Arnold et al., Phys. Rev. D79, (2009)

[2] COMPASS Collaboration, CERN-SPSC-2010-014, (2010)

[3] M. Anselmino et al., Phys. Rev. D75, (2007)

[4] M. Anselmino et al., Nucl. Phys. Proc. Suppl. 191, (2009)

[5] NA10, S. Falciano et al., Z. Phys. C31, 513 (1986)

[6] J. S. Conway et al., Phys. Rev. D39, 92 (1939)

[7] NA10, S. Guanziroli et al., Z. Phys. C37, 545, (1988)

[8] FNAL-E866/NuSea, L. Zhu et al., Phys. Rev. Lett. 99

[9] FNAL-E866/NuSea, L. Zhu et al., Phys. Rev. Lett. 102 